\title{
Safety and risk in practice
}

\author{
Edited by André F. Tempelaar \\ Het Eiland 7, 7271 BK Borculo, The Netherlands
}

\section{Nosocomial bloodstream infections: $20 \%$ of in-hospital deaths}

Nosocomial bloodstream infections represent a most important threat to patients admitted to hospital. It is believed that in the USA every year 62,500 hospitalized patients die of septicemia. Despite efforts for decades to prevent hospital-acquired infections the situation has not improved; indeed, both the overall incidence and the population-attributable risk of death among patients with nosocomial bloodstream infections has actually increased progressively during the last decade. This is the alarming conclusion of a 12-year retrospective study performed at the University of Iowa Hospitals and Clinics (Iowa City, USA).

In the University's main 902-bed hospital (with 213 beds reserved for all kind of critical care patients) a nosocomial infection surveillance programme has existed since 1976 . The study covers all the 260,834 patients (representing 2.4 million days of care) admitted to the hospital between 1980 and 1992. A total of 3464 nosocomial bloodstream infections (1.3\%) were identified in 3077 patients $(1.1 \%)$. A subdivision was made into primary bloodstream infections with an extracorporeal source and secondary bloodstream infections, caused by a diagnosed nosocomial infection at another site in the body (pneumonia, urinary tract infection).

The overall bloodstream infection rate increased from $0.7 \%$ in 1981 to $1.8 \%$ in 1992 , with a peak in $1991(2.1 \%)$. The proportion of primary bloodstream infections also increased progressively from $51 \%$ in 1981 to $71 \%$ in 1992 . At least $20 \%$ of the primary bloodstream infections was associated with intravascular device infections, particularly intravenous lines. Secondary bloodstream infections were mostly related to nosocomial lower respiratory tract infections, surgical wound infections and urinary tract infections. The number of infections with gram-positive cocci and yeasts had increased since 1981, while the number of infections with aerobic gram-negative pathogens had decreased.

The crude mortality of patients experiencing bloodstream infections was 39\% (1150) in the whole period, decreasing from $51 \%$ in 1981 to $32 \%$ in 1992 . The population-attributable mortality of bloodstream infections of all patients admitted had, however, increased from $0.36 \%$ in 1981 to $0.51 \%$ in 1992, while the population-attributable mortality of patients who did not experience bloodstream infections did not change during the sam? period.

In 1992 in the Iowa University Hospitals and Clinics one in every five in-hospital deaths was attributed to nosocomial bloodstream infections. Patients with a bloodstream infection had a 8-24 fold higher mortality risk ratio than did patients without bloodstream infections. 


\section{Reference}

[1] D. Pittet and R.P. Wenzel, Nosocomial bloodstream infections. Secular trends in rates, mortality, and contribution to total hospital deaths, Arch. Intern. Med. 155 (1995), 1177-1184.

\section{ICU-acquired infections: in some European countries it is better to avoid admission to Intensive Care!}

In Intensive Care Units (ICU), frequent utilization of invasive procedures and the serious and often critical condition of the patients admitted increase the risk of nosocomial infections. When one, however, makes comparisons across Europe, the risk of acquiring nosocomial infections after admittance at an ICU varies considerably. ICU's in Switzerland and in the Scandinavian countries have the lowest prevalence of ICU-acquired infections (9.7\% and 10.7\%). In Italian and Greek ICU's on the other hand the proportion of patients who experience a nosocomial infection is $31.6 \%$ and $30.5 \%$ respectively. The mortality rate among patients admitted to an ICU is found to be lower in Switzerland $(8.4 \%)$ and in Scandinavia $(9.8 \%)$ then in Greece $(28.5 \%)$ or Portugal $(23.9 \%)$.

These and other data emanate from the European Prevalence of Infection in Intensive Care Study (EPIC). In this investigation, a total of 1417 ICU's in 17 European countries, providing 10.038 case reports, participated voluntary in a 24-hour point-prevalence study. Excluding coronary care units, paediatric and special care infant units, about $40 \%$ of all European ICU's participated in the study.

Thirty percent of the admitted patients was older than 70 years; $14 \%$ of the patients remained in the ICU for more than three weeks. Antibiotics were prescribed to $62 \%$ of the patients admitted; $5.6 \%$ of the patients underwent selective decontamination of the digestive tract (most frequently in The Netherlands and in Austria: $14 \%$ and 15\% respectively).

Nosocomial infections were reported in $31 \%$ of all patients, comprising $21 \%$ ICU-acquired infections and $10 \%$ other hospital-acquired infections. Another $14 \%$ of the patients suffered community acquired infections. Pneumonia (47\%), lower respiratory tract infections (18\%), urinary tract infections (18\%) and bloodstream infections (12\%) were the infections most commonly acquired.

Major risk factors for ICU-acquired infections were found to be increasing length of stay, assisted ventilation, diagnosis of trauma, invasive procedures and stress ulcer prophylaxis.

Six weeks after the point-prevalence measurement the clinical outcome in 9273 patients was examined. The mean overall mortality rate was found to be $16.8 \%$. A high prevalence of nosocomial infections was correlated with high mortality. Mortality risk was highest in patients with ICU-acquired pneumonia, bloodstream infection or sepsis.

\section{Reference}

[1] J.-L. Vincent, D.J. Bihari, P.M. Suter et al., The prevalence of nosocomial infection in intensive care units in Europe, JAMA 274 (1995), 639-644.

\section{Stethoscopes and the noise of staphylococci}

Stethoscopes can be a vector for transmission of nosocomial infections, particularly staphylococci. In 1992 Breathnach et al. [1] cultivated positive cultures of staphylococci on $90 \%$ of the stethoscopes 
they investigated in a hospital. Nearly $20 \%$ of the positive cultures yielded S. aureus. Although cleaning the diaphragms with alcohol swabs decreased bacterial growth by $97 \%$, they also reported that doctors never (or only very occasionally) cleaned the diaphragms of their stethoscopes.

In neonatal intensive care units coagulase-negative staphylococci are the main cause of infection in very low birthweight infants. Wright et al. [2] investigated the cleaning practices of stethoscopes by doctors and nurses at a neonatal intensive care unit (in Leeds, UK). They could confirm the findings of Breathnach et al.: $55 \%$ of the nurses and $89 \%$ of the doctors never or only occasionally cleaned their stethoscopes and $67 \%$ of the stethoscopes used in the unit were contaminated by coagulase-negative staphylococci.

The problem was discussed with the nursing and medical staff. The nurses and doctors were asked to clean a stethoscope diaphragm with alcohol swabs each time they had used it, or when they borrowed it from another cot. It proved difficult to change the cleaning practices at once, but Wright et al. reported that after the intervention stethoscopes were cleaned at least once or twice a week. A repeated cultivation of swabs of the diaphragms after six weeks showed a decrease to $20 \%$ coagulase-negative staphylococci contaminated stethoscopes. As in the case of hand washing, there is a need for continuous instruction and audit to reduce nosocomial infections attributable to contaminated stethoscopes.

\section{References}

[1] A.S. Breathnach, D.R. Jenkins and S.J. Pedler, Stethoscopes as possible vectors of infection by staphylococci, Br. Med. J. 305 (1992), 1573-1574.

[2] I.M.R. Wright, H. Orr and C. Porter, Stethoscope contamination in the neonatal intensive care unit, J. Hosp. Infect. 29 (1995), 65-68.

\section{Late radiation neuropathy of the brachial plexus: should the management of early stage breast cancer be revised?}

The aim of radiotherapy is optimal tumour control with minimal damage to tissue (Olsen et al. [1]). Radiation-induced brachial plexopathy is a serious late complication of radiation therapy in the treatment of early stage breast cancer, and can cause permanent disability. The median period elapsing between radiation therapy and the first sign of neuropathy was found to be 1.5-77 months.

Susan Pierce et al. [2] (Harvard Medical School, Boston, USA) found a 1.8\% incidence of brachial plexopathy in a group of 1117 women who had undergone supraclavicular irradiation with or without axillary irradiation. The majority of the 20 identified cases recovered.

However the Danish Breast Cancer Cooperative Group found a 14\% complication rate of brachial neuropathy in a group of 128 recurrence-free breast cancer patients (with a median follow-up of 50 months after radiation therapy). Disabling plexopathy was found to $5 \%$ of the treated women. Clinical manifestations were paraesthesia (100\%), hypaesthesia (74\%), weakness $(58 \%)$, decreased muscle stretch reflexes (47\%) and pain (47\%). Axillary irradiation and high dose irradiation (fraction size $>2$ Gy and total dose $>50$ Gy) were correlated with a higher risk of plexopathy. The Danish and the American investigators found also a higher risk of late radiation plexopathy in young women and in cases receiving adjuvant treatment with chemotherapy.

In the UK a pressure group of 800 women who claim iatrogenic damage by radiation therapy has secured the attention of the medical authorities (Radiotherapy Action Group Exposure; RAGE). 
The Royal College of Radiologists has commissioned an independent survey; 556 women who were members of RAGE and who claimed nerve damage after radiotherapy were asked to participate in the investigation. All 556 women had been treated between 1980 and 1993 at one of 15 centres in England and Wales. During this period about 65,000 women with breast cancer had been treated in these centres with radiotherapy, but data on the total incidence of the complication were not available.

Against the advice of their solicitors, 126 women agreed to examination of their medical records. The two senior oncologists who performed the investigation found 48 cases of probable brachial plexus neuropathy due to radiotherapy. Most of the patients with neuropathy (41) had been treated before 1986, and the higher doses of radiation to the axilla region which were customary up to that date were seen as a likely causal factor. The main blame was laid on the movements of the patients' arm occurring between radiotherapy to the breast and radiotherapy to the axillary region. Patients who had moved their arms in this way developed neuropathy three times more often (72\%) as compared with patients who had not moved (24\%).

Oncologist Dr. Margaret Spittle proposes revision of the management of early breast cancer treatment [3]. The impact of radiotherapy on long-term survival is disputable, since a 5\% reduction in death from breast cancer seems to be counterbalanced by an increase in death from other causes. Despite the dramatic improvement of radiotherapy, adjuvant chemotherapy, hormone therapy and improved surgical management of the axilla can possible make the need for radiotherapy in the axillary region superfluous.

\section{References}

[1] N.K. Olsen, P. Pfeiffer, L. Johannsen et al., Radiation-induced brachial plexopathy: neurological follow-up in 161 recurrence-free breast cancer patients, Int. J. Radiation Oncology Biol. Phys. 26 (1993), 43-49.

[2] S.M. Pierce, A. Recht, T.I. Lingos et al., Long-term radiation complications following conservative surgery (CS) and radiation therapy (RT) in patients with early stage breast cancer, Int. J. Radiation Oncology Biol. Phys. 23 (1992), 915-923.

[3] M.F. Spittle, Brachial plexus neuropathy after radiotherapy for breast cancer, Br. Med. J. 311 (1995), 1516-1517.

\section{Pain after axillary dissection for early stage breast cancer: not always a nerve lesion}

Dissection of the lymph nodes in the axillary region is a standard treatment of early stage breast cancer. Postoperative pain and hypaesthesia are often caused by cutting the sensory branches of the $n$. intercostobrachialis. Particularly when dissecting the nodes in the median axillary top region, damage to this nerve is nearly inevitable. About one third of the women with a median top dissection complains of postoperative pain, two thirds have sensations of hypaesthesia and one to three of the operated women has found themselves more or less disabled (Van Dam et al. [1]).

The Dutch surgeon R.M.H. Roumen has now drawn attention to another complication causing shoulder pain after axillary dissection [2]. He found a painful tendinitis of the short biceps tendon in about $5 \%$ of the patients after the dissection. The pain sensations resembled those of intercostobrachialis neuropathy.

The tendinitis is probably caused by overloading of the biceps tendon, because of the altered anatomy after dissection. The complication can easily be treated with local injection of a combination of long-acting local anaesthetic and a depot steroid in the tendon near the coracoid processus.

The complaints from intercostobrachial neuropathy can mostly be prevented by leaving the third median top level untouched, dissecting only the first and second levels of lymph nodes in the axillary 
region with special care for the nerve. Siegel et al. [3] recorded only a $1.5 \%$ neuropathy rate when operating in this way, and the risk of local recurrence was no greater.

\section{References}

[1] M.S.W. van Dam, A. Hennipman, J.Th.C.M. de Kruif et al., Complicaties na okselkliertoilet wegens mammacarcinoom. (Postoperative pain after axillary dissection for breast cancer), Ned Tijdschr Geneeskd 137 (1993), 2395-2398.

[2] R.M.H. Roumen, Tendinitis van de korte bicepspees na okselklierdissectie (Tendinitis of the short biceps tendon after axillary lymph node dissection), Ned Tijdschr Geneeskd 140 (1996), 271-272.

[3] B.M. Siegel, K.A. Mayzel, S.M. Love et al., Level I and II axillary dissection in the treatment of early-stage breast cancer. An analysis of 259 consecutive patients, Arch. Surg. 125 (1990), 1144-1147.

\section{Survival from breast cancer: choose a surgeon with much experience, working in a multidisciplinary setting}

Surgeons who see more than 30 new cases of breast cancer per year and who have a full range of treatment options available within a multidisciplinary setting have better survival prognosis for their patients. This is the conclusion of a study analysing cancer registry data from 12,861 women treated for breast cancer in Yorkshire (UK) between 1979 and 1988. Richard Sainsbury et al. [1] found considerable outcome variation between 180 consultants managing the treatment of women with breast cancer. Patients treated with chemotherapy and hormone therapy adjuvant to surgery had a better prognosis, explaining $26 \%$ of the statistical variation. Surgeons with higher caseloads (more than 30 new cases per year) achieved significantly better survival results than surgeons with caseloads between 10 and 29 patients per year. It turned out that clinicians with higher caseloads more often prescribed adjuvant chemotherapy or hormone therapy and used higher doses. A multidisciplinary approach (availability of the whole range of treatment options) was also correlated with better survival results.

If the management routines of the group of surgeons with the best clinical outcome were to become a golden standard for treatment by all clinicians dealing with this condition, the mean survival after five years would increase by some 4-5\% (corrected for age, extent and grade of disease).

In Yorkshire $55 \%$ of patients were treated by surgeons managing less than 30 patients per year and $10 \%$ by surgeons managing less than 10 patients per year. About $30 \%$ of the women were treated by eight surgeons, managing more than 50 patients per year. Over the period of the study there was a trend for more patients to be treated by clinicians having higher caseloads.

\section{Reference}

[1] R. Sainsbury, B. Haward, L. Rider et al., Influence of clinician workload and patterns of treatment on survival from breast cancer, Lancet 345 (1995), 1265-1270.

\section{Medication errors in hospitals: a ward-based dispensing system compared with a unit dose dispensing system}

A medication error can be defined as a discrepancy between the medication prescribed and the medication actually administered. In the 1960s, in hospitals in the United States and in the United 
Kingdom, nurses commonly transcribed medication orders from physicians into lists of doses to be administered. In both countries medication error rates up to $15 \%$ were identified. Dispensing systems have since changed, but in a different way.

Most UK hospitals today have a ward-based dispensing system with a stock of frequently used drugs kept on the wards, supervising pharmacists visiting the wards daily, using combined drug prescription sheets and medication administration records which also provide the recent drug history of the patient.

In the USA, by contrast, most hospitals have chosen for an "incomplete" unit dose dispensing system with individually wrapped medications but with the nurse free to determine how much must be administered. The pharmacists generate drug profiles of all patients every 24 hours (often computerized) from which medication administration records are made, and these are used by nurses to select the patient's medication. In US hospitals little or no drug stock is kept in the patient wards, though there may be satellite pharmacies supplying specialized care units.

London pharmacist Bryony Dean et al. compared medication errors in wards with a workload involving much oral medication in two large university hospitals in the UK and the USA [1].

In the US hospital, 6.9\% medication errors were identified in a total of 919 instances of medication observed. In the UK hospital 3.0\% medication errors were found in 2756 instances of administration.

In the US hospital, incorrect dosage, use of an unordered dose and omission of the ordered dose were the most frequent errors. In the UK hospital omission of ordered dose comprised $58 \%$ of the medication errors. In both hospitals the most likely cause of the errors observed was incorrect selection or preparation by the nurse (52\% in the US hospital and $40 \%$ in the UK hospital). In the US hospital lack of clarity in the prescription or the use of incorrect medication administration records (37\%) was a second important cause of error, whereas in the UK hospital unavailability of the ordered drugs on the ward $(39 \%)$ was a prominent secondary cause of medication error.

The differences between the two dispensing systems explains in part the differing outcome. Dean et al. concluded that strong points in one system turned out to be weak points in the other system. In the US dispensing system the adequate availability of the drugs needed was a strong point, whereas this was a weak point in the UK system. On the other hand the prescribing and transcription process, which is weak in the US system (readily leading to commission of errors), is a strong point of the UK system, where physicians write their orders on a medication chart. Oral orders from physicians to nurses are not allowed. In the British system pharmacists visit the wards daily, supervising the dispensing system. The US computer-generated medication profiles were useful, but $37 \%$ of the errors observed were due to incorrect medication administration records, resulting in the omission of ordered drugs or the administration of drugs that had been discontinued. In both systems the poor handwriting of prescribers was a major problem.

\section{Reference}

[1] B.S. Dean, E.L. Allan, N.D. Barber et al., Comparison of medication errors in an American and a British hospital, Am. J. Health-Syst. Pharm. 52 (1995), 2543-2549.

\section{Asthmatic patients and irregular use of major tranquillisers: an increased risk of death from asthma}

The association between risk of death and near death from asthma and the use of drugs by asthma patients is subject of research at the McGill University in Montreal, Canada. Joseph et al. investigated the use of drugs for coexistent psychiatric disorders in patients with bronchial asthma [1]. 
Patients with both asthma and psychiatric disorders are more at risk for sub-optimal treatment of both diseases. Aggravation of the clinical condition either due to the patient's noncompliance or the abuse of prescribed medications is a general concern in the management of treatment. Physicians also have to take into account the fact that the drugs prescribed for treating asthma can interfere adversely with the clinical condition of psychosis and vice versa. Some drugs used for asthma treatment have effects on the mental state of the patient (systemic corticosteroids, $\beta$-agonists), while major tranquillisers can cause sedation with respiratory dysfunction and other autonomic nervous effects.

Joseph et al. conducted a population-based study to quantify the increased risk of death or near death from asthma among asthma patients using major tranquillisers. Using data from the Saskatchewan Asthma Epidemiology Project they analyzed a cohort of 12,301 patients between 5 and 54 years using anti-asthmatic drugs. They concluded that asthma patients using major tranquillisers in the preceding 12 month period were at a 3.2 (95\% confidence interval 1.4 to 7.5 ) times greater risk of death or near death from asthma than were asthma patients who did not use major tranquillisers. Whereas continuous use of major tranquillisers was not associated with an increased risk, asthma patients who had recently discontinued the use of major tranquillisers were particularly at risk (relative risk 6.6; $95 \%$ confidence interval 2.5 to 17.6 ).

The risk of death from asthma among asthma patients using antidepressants, antihypertensives or hypoglycaemic agents was not significantly higher. But asthma patients using sedatives and minor tranquillisers were 1.9 (95\% confidence interval 1.2 to 3.1) times higher at risk.

The Canadian group urges physicians to consider a history of use of major tranquillisers as a marker for identifying patients having a higher risk of serious complications in asthma.

\section{Reference}

[1] K.S. Joseph, L. Blais, P. Ernst et al., Increased morbidity and mortality related to asthma among asthmatic patients who use major tranquillisers, Br. Med. J. 312 (1996), 79-82.

\section{Prevention of postoperative delirium}

Postoperative delirium is defined as an acute change in cognitive status characterized by fluctuating consciousness and inattention occurring within 30 days after an operation. Dyer et al. found 80 primary data collection studies in the English language literature between 1966 and 1992 providing incidence estimates and risk factor analysis of postoperative delirium [1]. They analyzed 26 articles meeting their criteria of validity (diagnostic methods, inclusion and exclusion criteria, data handling), including 15 studies relating to cardiac surgery. Seventeen other studies were acceptable for risk analysis. The incidence of postoperative delirium ranged from $0 \%$ to $73.5 \%$ with an overall incidence of $37 \%$. The wide variation was merely due to the way definitions were used and the methods of study adopted. The diagnosis of postoperative delirium is often missed. In five studies using self-reporting by patients the overall incidence was $58 \%$. And in another study with psychiatric follow up, it was determined that physicians and nurses missed $28 \%$ of the diagnoses.

Older patients have a higher risk of postoperative delirium than young patients. However, other risk factors include pre-existent cognitive impairment, depression, postoperative use of five or more different drugs and preoperative use of anticholinergic drugs. No difference was found between cases which had undergone spinal as compared to general anaesthesia. 
The risk of postoperative confusion could be decreased by providing adequate psychological support before and after the operation, allowing the patients to express their fears. An interventional study on the effects of attendance by a geriatrician showed that it resulted in a decrease in postoperative morbidity and length of stay in hospital.

The authors of this review concluded that postoperative confusion state is a more common postoperative problem than sometimes believed, but that its incidence can be reduced by rigorous pre- and postoperative management programmes.

\section{Reference}

[1] C.B. Dyer, C.M. Ashton and T.A. Teasdale, Postoperative delirium: a review of 80 primary data-collection studies, Arch. Intern. Med. 155 (1995), 461-465.

\section{The problem of malfunctioning doctors demands a fundamental approach}

Chief-editor Richard Smith of British Medical Journal has roundly condemned the report of the British Department of Health about the problem of poorly performing doctors [1, 2]. In his view the facile conclusion of this poorly written document, to the effect that culture within medicine and within the National Health System is wrong, is not founded on data. The problem is not explored and indeed no references are provided to worldwide experience with the problem of poor medical performance. The report, a governmental reaction to media reports about incompetent doctors in 1993 , is no more than a defensive cover for future scandals "... so that the government can say that it is doing something".

The real problem, Richard Smith points out, is "the culture in which medical students have been humiliated for not knowing, and so have learnt to hide their ignorance, in which junior doctors are reluctant to call their seniors, and so take on more than they should, in which it is seen as weakness to admit anxieties or shortcomings, in which errors are seen as unacceptable and so are hidden, and in which all doctors find it hard to confront colleagues who are performing poorly".

The disappointing governmental report will not change this culture by contracts, circulars, discussion in august bodies and legislation. There is need for more fundamental approach of the problem, creating a culture of continuous quality improvement. A culture where a defect is considered a matter demanding the most serious attention. A combination of bottom-up processes and leadership, motivating people to do what needs to be done. A culture starting at the base of medical education.

\section{References}

[1] Department of Health, Review of guidance on doctor's performance. Maintaining medical excellence. DoH, London, 1995.

[2] R. Smith, British government's proposals on poorly performing doctors, Br. Med. J. 311 (1995), 402.

\section{Preventing malpractice claims in the Ottoman Empire}

To avoid experiencing claims relating to adverse outcomes of hazardous medical treatment, physicians in the Ottoman Empire could employ a legally valid form of informed consent. Dr. Kamel 
Ajlouni [1] of the Faculty of Medicine of the Jordan University of Science and Technology has drawn attention to a documented case of legal informed medical consent, signed before the Tripoli-Syria Holy Sharia Council in November 10, 1677 (12 Shaban 1088).

The attest describes an agreement about the hiring and engagement of the surgeon Nicola to reduce a hernia and provide postoperative treatment in a man named Ya'coub, in return for a fee of ten piasters. The text of the document tells how in the presence of witnesses the surgeon was absolved of responsibility, claims and blood money, if following due treatment the patient should die as a result of fate and God's divine decree.

\section{Reference}

[1] K.M. Ajlouni, History of informed medical consent, Lancet 346 (1995), 980. 